\title{
Network-timing-dependent plasticity
}

\author{
Vincent Delattre ${ }^{1}$, Daniel Keller ${ }^{2}$, Matthew Perich ${ }^{2,3}$, Henry Markram ${ }^{1,2}$ and \\ Eilif B. Muller ${ }^{2 *}$
}

${ }^{1}$ Laboratory of Neural Microcircuitry, Brain and Mind Institute, École Polytechnique Fédérale de Lausanne, Lausanne, Switzerland, ${ }^{2}$ Center for Brain Simulation, École Polytechnique Fédérale de Lausanne, Geneva, Switzerland, ${ }^{3}$ Department of Biomedical Engineering, Northwestern University, Evanston, IL, USA

\section{OPEN ACCESS}

Edited by:

Dion Dickman,

University of Southern California, USA

Reviewed by:

Annalisa Scimemi,

The State University of New York

at Albany, USA

Lisa Mapelli,

University of Pavia, Italy

${ }^{*}$ Correspondence:

Eilif $B$. Muller,

Center for Brain Simulation, École

Polytechnique Fédérale de Lausanne,

Biotech Campus, Chemin des

Mines 9, CH-1202 Geneva,

Switzerland

eilif.mueller@epfl.ch

Received: 17 March 2015

Accepted: 22 May 2015

Published: 09 June 2015

Citation:

Delattre V, Keller D, Perich M, Markram H and Muller EB (2015) Network-timing-dependent plasticity.

Front. Cell. Neurosci. 9:220.

doi: 10.3389/fncel.2015.00220
Bursts of activity in networks of neurons are thought to convey salient information and drive synaptic plasticity. Here we report that network bursts also exert a profound effect on Spike-Timing-Dependent Plasticity (STDP). In acute slices of juvenile rat somatosensory cortex we paired a network burst, which alone induced long-term depression (LTD), with STDP-induced long-term potentiation (LTP) and LTD. We observed that STDP-induced LTP was either unaffected, blocked or flipped into LTD by the network burst, and that STDP-induced LTD was either saturated or flipped into LTP, depending on the relative timing of the network burst with respect to spike coincidences of the STDP event. We hypothesized that network bursts flip STDP-induced LTP to LTD by depleting resources needed for LTP and therefore developed a resourcedependent STDP learning rule. In a model neural network under the influence of the proposed resource-dependent STDP rule, we found that excitatory synaptic coupling was homeostatically regulated to produce power law distributed burst amplitudes reflecting self-organized criticality, a state that ensures optimal information coding.

Keywords: synaptic plasticity, patch-clamp, acute brain slices, somatosensory cortex, STDP, self-organized criticality, neural networks simulations

\section{Introduction}

Periods of synchronous neuronal firing, or bursts of action potentials (APs) in populations of neurons, are ubiquitous in the central nervous system. Bursts can induce long-lasting changes in synaptic efficacy depending on the frequency of bursting, with long-term depression (LTD) being induced by low frequency bursts and long-term potentiation (LTP) being induced at higher frequencies (Bliss and Lomo, 1973; Lynch et al., 1983; Stanton and Sejnowski, 1989; Dudek and Bear, 1992) with notable exceptions (Coesmans et al., 2004). This phenomenon led to the wellknown Bienenstock Cooper and Munro (BCM) model of synaptic plasticity (Bienenstock et al., 1982). The relative timing of single spikes generated in connected pairs of neurons can also induce LTP and LTD (Markram et al., 1997; Bi and Poo, 1998), which has led to the well-known SpikeTiming-Dependent Plasticity (STDP) model of synaptic plasticity (Markram et al., 2011). The manner in which these two induction protocols for synaptic plasticity interact is unclear, and it remains to be seen if they can be unified under a common mechanism.

According to the so-called calcium hypothesis, synaptic changes are thought to be determined by the magnitude and time-course of the transient influx of calcium into the synaptic spine induced by pre- and post-synaptic spiking (Bear et al., 1987; Shouval and Kalantzis, 2005; Nevian and Sakmann, 2006; Graupner and Brunel, 2010, 2012). Large calcium influxes are thought to induce potentiation, whereas moderate and prolonged calcium influxes are thought to induce depression (Bienenstock et al., 1982; Nevian and Sakmann, 2006). A network burst induced transient 
reduction in extracellular calcium would reduce the magnitude of calcium influx into the spine approximately proportionally (Egelman and Montague, 1999; Wiest et al., 2000), and could subsequently alter the outcome of plasticity. In particular, spiking motifs yielding LTP could instead yield LTD when embedded in a network burst.

This shifting of the direction of plasticity in bursting networks toward LTD is an interesting observation, as it is a possible mechanism for counteracting run-away potentiation in networks of neurons with on-going synaptic plasticity. Increases in synaptic coupling between excitatory neurons are known to induce a transition to bursting activity regimes, as has been reported in previous theoretical studies (Tsodyks et al., 2000; Kudela et al., 2003) and under pathological experimental conditions where synaptic up-scaling was induced by activity deprivation (Trasande and Ramirez, 2007). While homeostatic mechanisms have been proposed to down-regulate synaptic strengths if neuronal firing rates become excessive (Turrigiano et al., 1998; Trasande and Ramirez, 2007), such mechanisms have been shown to be insufficient to maintain network stability in simulations of networks of neurons incorporating empirically constrained STDP models at excitatory synapses (Zenke et al., 2013). One important reason for this is that such mechanisms are insensitive to the transition to the network bursting state, which occurs with only minor changes in neuronal firing rates. The proposed interplay between network bursting activity and STDP could provide negative feedback allowing fine homeostatic control to be maintained in the presence of on-going synaptic plasticity, and thus to maintain states of criticality observed in cortical networks (Beggs and Plenz, 2003; Priesemann et al., 2009, 2013).

To gain insight into the proposed interaction of STDP and network bursting activity, we investigated in vitro the effect of precisely timed network bursts on STDP at excitatory synaptic inputs to layer 5 pyramidal neurons where the STDP phenomenon was first reported (Markram et al., 1997). STDP protocols known to induce LTP and LTD were applied, and network bursts were induced at precise timings before, during, or after the STDP pairing protocols using the electrodes of a multielectrode array (MEA) located in layer 5. The pairing of STDP events with network bursts can influence the plasticity outcome by altering the timing relationship in the pre-post spike motif due to the additional spikes, and by changes in context due to the network burst (such as voltage, competition for resources, etc.). To separate the former effects from the latter, we performed burst-spike-substitution (BSS) experiments whereby the MEA burst was replaced with an excitatory postsynaptic potential (EPSP) paired with a simultaneous post-synaptic AP.

Our main experimental finding is that certain specific timings of network bursts relative to the STDP events can induce flips of LTD into LTP and LTP into LTD, which cannot be accounted for by the BSS protocols, and thus on pre-post spiking alone. We propose that the observed flips are manifestations of positive and negative synaptic cooperativity, respectively, for which a number of mechanisms have been proposed. We hypothesize that negative cooperativity could be due to the depletion of critical resources needed for LTP, perhaps through the depletion of an intracellular messenger (Fonseca et al., 2004), or the transient reduction of extracellular calcium at synaptic junctions immediately following network bursting (Egelman and Montague, 1999; Wiest et al., 2000).

We further hypothesize that the observed negative cooperativity could have an important role in the maintenance of the excitation-inhibition balance and of network criticality in the presence of on-going synaptic plasticity. To evaluate this hypothesis, we employ simulations of networks of neurons incorporating an empirically constrained STDP rule (Morrison et al., 2007), and augment it with a resource depletion term implementing a shift of STDP outcomes from LTP to LTD when embedded in a network burst. Networks including the resource depletion term are found to induce a transition to a state of criticality in the network (Beggs and Plenz, 2003; Priesemann et al., 2009, 2013). The proposed resource-dependent interaction between network activity and STDP therefore represents a novel mechanism for the homeostatic regulation of the network activity regime.

\section{Materials and Methods}

\section{Electrophysiology}

In accordance with the Swiss national and institutional guidelines, $300 \mu \mathrm{m}$ thick sagittal brain slices were prepared from somatosensory cortex of postnatal days 13-17 Wistar rats of either sex in iced artificial cerebrospinal fluid (ACSF) containing (in mM) $125 \mathrm{NaCl}, 2.5 \mathrm{KCl}, 25 \mathrm{D}$-glucose, $25 \mathrm{NaHCO}_{3}, 1.25$ $\mathrm{NaH}_{2} \mathrm{PO}_{4}, 2 \mathrm{CaCl}_{2}$, and $1 \mathrm{MgCl}_{2}$; all chemicals from SigmaAldrich (St. Louis, MO, USA or Merck, Darmstadt, Germany), using a HR2 vibratome (Sigmann Elektronik, Heidelberg, Germany). The primary somatosensory cortex was manually dissected and isolated to obtain rectangular slices of 5-7 mm width and containing the neocortex in its entire height. Optimal slices, with apical cell dendrites running parallel to the slice surface, were selected for recordings. Slices were incubated at $22^{\circ} \mathrm{C}$ for $30-60$ min until mounting in the recording chamber. Slices were mounted on a 3D-MEA with 60 pyramidal platinum electrodes (electrode basis: $40 \mu \mathrm{m} \times 40 \mu \mathrm{m}$, electrode height: 50$70 \mu \mathrm{m}$, electrode interspacing: $200 \mu \mathrm{m}$; Qwane Bioscience SA, Lausanne, Switzerland) after evaporation of a mounting solution of $0.14 \mathrm{mg} / \mathrm{L}$ nitrocellulose in an ethanol (99\%) - methanol (1\%) mixture. Cells were visualized by infrared differential interference contrast video microscopy using a camera (VX 55, Till Photonics, Gräfelfing, Germany) mounted on an upright microscope (BX 51WI, Olympus, FI, Japan) fitted with a $40 \times$ objective (LUMPLAN, Olympus). Whole-cell recordings were performed using Axopatch 200B amplifiers (Molecular Devices, Union City, CA, USA). Data acquisition, sampled at $5-10 \mathrm{kHz}$, was performed via an ITC-18 board (Instrutech Co, Port Washington, NY, USA), connected to a computer running IgorPro (Wavemetrics, Portland, OR, USA). The voltage signal was filtered with a $2 \mathrm{kHz}$ Bessel filter. Multiple somatic whole cell recordings ( $1-3$ cells simultaneously) were performed using patch pipettes pulled with a P-97 Flaming/Brown micropipette puller (Sutter Instruments Co, Novato, CA, USA) with an initial 
resistance of 8-10 M $\Omega$. Patch pipettes were filled with standard intracellular solution (ICS) containing (in $\mathrm{mM}$ ): $110 \mathrm{~K}$-gluconate, $10 \mathrm{KCl}, 4$ ATP-Mg, 10 phosphocreatine, 0.3 GTP, $10 \mathrm{~N}-2$ hydroxyethylpiperazine- $N^{\prime}$-2-ethanesulfonic acid $(\mathrm{pH} 7.3)$, and $0.5 \%$ biocytin. Recordings were not corrected for the liquid junction potential between ACSF and ICS $(-14 \mathrm{mV})$. Variation in the cell input resistance was measured from beginning to end of the experiment, and all cells having a change in input resistance greater than $33 \%$ were excluded. Cell access resistance was typically less than $20 \mathrm{M} \Omega$.

\section{Electrical Stimulation}

A STDP protocol known to induce LTP (the STDP ${ }^{+}$event) was applied as a $50 \mathrm{~Hz}$ train of three APs with a single evoked EPSP $10 \mathrm{~ms}$ earlier (Nevian and Sakmann, 2006; Figure 1B). A STDP protocol known to induce LTD (the STDP ${ }^{-}$event) was applied as a $50 \mathrm{~Hz}$ train of three APs with a single evoked EPSP $10 \mathrm{~ms}$ later (Nevian and Sakmann, 2006; Figure 1C). EPSPs were evoked by extracellular stimulation using an extracellular pipette located near the basal dendrites of the patched-cell, and paired with APs evoked with supra-threshold intracellular current injection, as previously described (Nevian and Sakmann, 2006). Pairings were repeated 60 times at a frequency of $0.1 \mathrm{~Hz}$. EPSPs were monitored at a frequency of $0.1 \mathrm{~Hz}$, for $10 \mathrm{~min}$ prior to pairing to record the baseline and for more than $1 \mathrm{~h}$ post pairing. Network bursts were evoked by extra-cellular electrical stimulation of layer 5 (STG2008 stimulator, Multi Channel System, Reutlingen, Germany) using a 3D-MEA (electrode basis: $40 \mu \mathrm{m} \times 40 \mu \mathrm{m}$, electrode height: 50-70 $\mu \mathrm{m}$, inter-electrode spacing: $200 \mu \mathrm{m}$; Qwane Bioscience SA, Lausanne, Switzerland). Stimulation strength was tuned to trigger a single AP in every patched cell (1-2 V biphasic pulses; $1 \mathrm{~ms}$ duration in each polarity). On average, the network burst failed to evoke a spike in $6.8 \%$ of the cases, triggered a single spike in $89.2 \%$ of the cases, and two spikes in $4 \%$ of the cases (Supplementary Figure S1). We never observed a network burst causing more than two spikes in the patched cells. The latency of the first evoked spike was $3.7 \pm 0.2 \mathrm{~ms}$ (range $1.4-10.8 \mathrm{~ms}, n=3960$ network bursts recorded in 66 cells). Due to the symmetry of the MEA evoked network burst, pre-synaptic spiking in excitatory cells during the burst is assumed to mirror post-synaptic spiking. The time interval $\Delta T$ between EPSP and the network burst was defined as the time between EPSP digital trigger and the network burst digital trigger.

Bursts were evoked before $(-20 \mathrm{~ms})$, simultaneous to $(0 \mathrm{~ms})$ or at the end of $(50 \mathrm{~ms})$ an $\mathrm{STDP}^{+}$event, and at the beginning of $(-50 \mathrm{~ms})$, simultaneous to $(0 \mathrm{~ms})$, or after $(20 \mathrm{~ms})$ an $\mathrm{STDP}^{-}$ event. The timing of the burst with respect to the STDP ${ }^{-}$protocol to was chosen to exactly mirror all tested protocols for LTP. The combined burst-STDP event pairing was applied at a frequency of $0.1 \mathrm{~Hz}$.

Burst-spike-substitution experiments replaced the network burst with an EPSP paired with a simultaneous post-synaptic AP for burst-STDP pairings, to mirror the pre- and postsynaptic spiking as seen by a synapse during a burst-STDP event (assuming the predominant case above that bursts trigger a single pre- and post-synaptic spike), but without the network context.

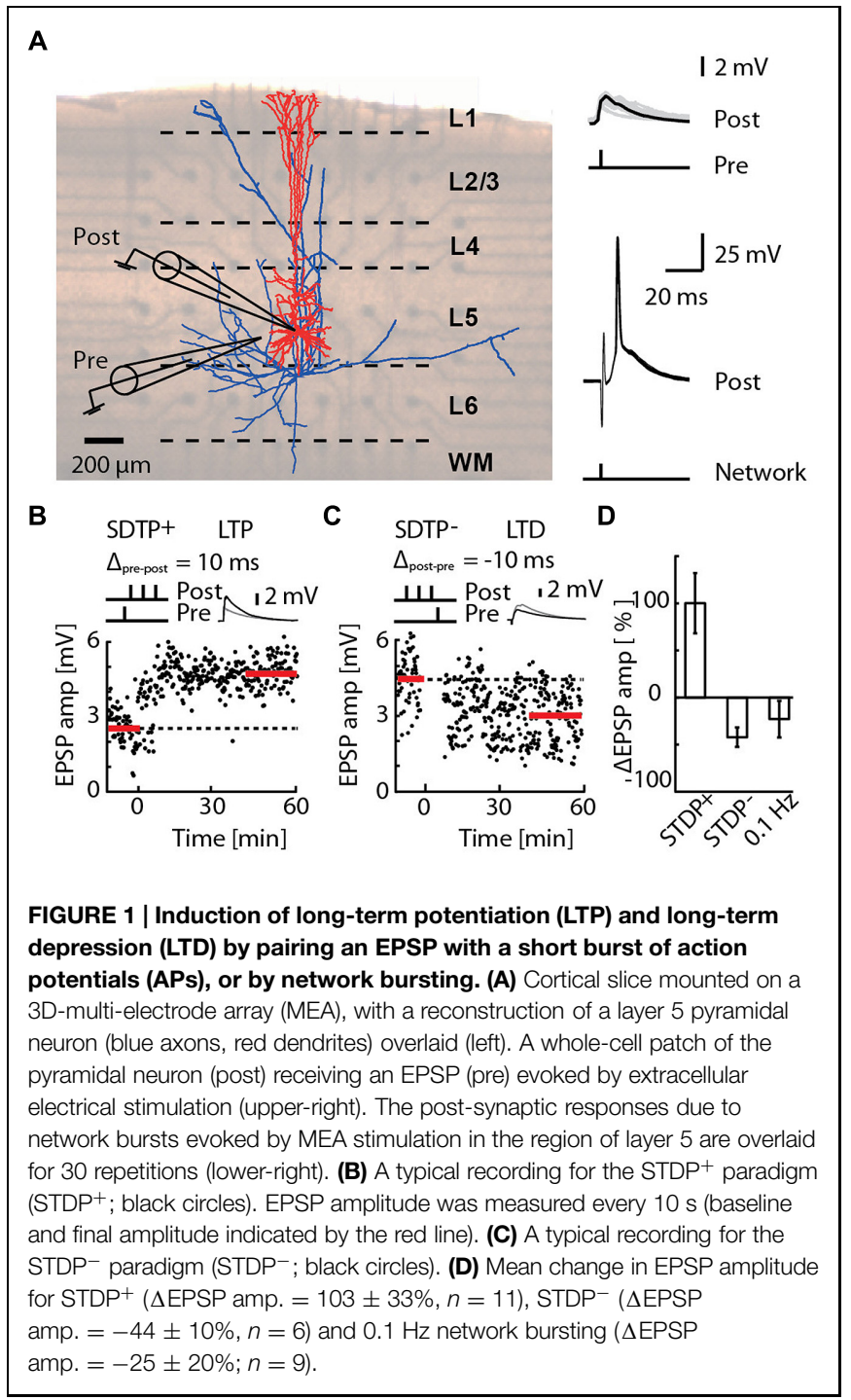

\section{Experimental Data Analysis and Statistics}

Experimental data analysis was performed in Matlab (The MathWorks, Inc., Natick, MA, USA) with custom scripts. EPSP amplitude was monitored for an hour and $20 \mathrm{~min}$. Baseline EPSP was acquired over the first $10 \mathrm{~min}$, followed by $10 \mathrm{~min}$ of pairing, as described above. The final EPSP amplitude was averaged over the last 20 min of recordings. EPSP failure or EPSPs that caused the cell to spike were excluded from the analysis. However, if after the pairing, a cell fired $100 \%$ of the time following the EPSP onset within an averaging time window (20 repetitions), we assumed a strong potentiation to have occurred and set the synaptic gain to a value of 5 for this time period. Data are presented as the mean \pm SEM. Paired Student's $t$-tests were applied as statistical tests, and statistical significance was asserted for: ${ }^{*} p<0.05$; ${ }^{* *} p<0.01 ;{ }^{* * *} p<0.001$.

\section{Network Simulations}

We simulated a network of 1000 integrate-and-fire (IF) neurons (of which $80 \%$ are excitatory and $20 \%$ inhibitory) 
arranged on a $10 \times 10 \times 10$ lattice, corresponding to a $200 \mu \mathrm{m} \times 200 \mu \mathrm{m} \times 200 \mu \mathrm{m}$ volume of cortex in an active state. Neuron parameters were fit to publicly available Hodgkin-Huxley type neuron models (Destexhe et al., 1998) for f-vs-I curves and noise current injections. Excitatory neurons contained a spike triggered conductance inducing spike-frequency adaptation (Muller et al., 2007). Each neuron had 1000 excitatory (AMPA) and 250 inhibitory $\left(\mathrm{GABA}_{\mathrm{A}}\right)$ conductance-based synapses with a peak conductance of $2 \mathrm{nS}$ (except plastic synapses) and time constants of $1.5 \mathrm{~ms}$ and $10 \mathrm{~ms}$, respectively. Consistent with anatomy, $10-20 \%$ of the synaptic inputs originated from neurons inside the network (140 exc. $\rightarrow$ exc., 200 exc. $\rightarrow$ inh., 50 inh. $\rightarrow$ exc., 50 inh. $\rightarrow$ inh.), and delays were computed as $d \beta(1+\xi)$, where $d$ is the distance, $\beta=0.25 \mathrm{~ms} /$ unit lattice and $\xi$ is a random number drawn from an exponential distribution with mean of 0.2. Extrinsic input was modeled by Poisson processes with firing rates parameterized separately for excitatory and inhibitory input, 6 and $10.5 \mathrm{~Hz}$, respectively. These rates were determined numerically to be consistent with excitatory and inhibitory model neuron firing rates resulting from application of exclusively Poissonian input at these rates at all synapses. Neuronal properties in the network are consistent with the "high-conductance state" (Destexhe et al., 2003). We used the Power-law STDP rule (Morrison et al., 2007) parameterized for cortical (Froemke and Dan, 2002) conductance-based synapses to achieve a mean of $\sim 1.9 \mathrm{nS}$ under extrinsic input alone as follows: $\tau_{+}=14 \mathrm{~ms} ; \tau_{-}=34 \mathrm{~ms}$; $w_{0}=4.29 \times 10^{-2} \mathrm{nS} ; \mu=0.4 ; \lambda=0.1$; and $\alpha=4.8 \times 10^{-2}$. For the computation of STDP time differences, connection transmission delays were treated as half axonal and half dendritic. Where stated, the STDP rule was augmented with a model for activity-dependent resource availability as described in the main text. The network and neuron models were implemented using the PyNN modeling language (Davison et al., 2009) with the NEURON simulator backend (Hines and Carnevale, 1997) and are publicly available at: https://neuralensemble.org/svn/PyNN/trunk/examples/iaf_sfa relref/

\section{Resource Dependent STDP}

To implement the hypothesized effects of resource depletion, such as extracellular calcium, on STDP as a mechanism to flip LTP into LTD, we added a resource depletion term to a standard STDP learning rule (Morrison et al., 2007) in the network model. We modeled resource depletion caused by network activity by assuming the equilibrated resource availability for any fixed average network firing rate, $\alpha$, has the form $\eta_{0}(\alpha)=$ $(1+\alpha / k)^{-1}$, where $k$ is the depletion rate constant ( $k$ was assigned to $20 \mathrm{~Hz}$ to allow for a $50 \%$ resource depletion during sustained $20 \mathrm{~Hz}$ network activity). The dynamic resource availability $\eta(t)$ was then computed by low pass-filtering $\eta_{0}(\alpha(t))$ as follows:

$$
\frac{d}{d t} \eta(t)=\frac{\eta_{0}(\alpha(t))-\eta(t)}{\eta_{0}(\alpha(t)) \cdot \tau_{\eta}},
$$

where $\alpha(t)$ is a continuous estimator of the average networking firing rate (low-pass filtered network spiking with a filter time constant $\tau_{\alpha}=2.5 \mathrm{~ms}$ and normalized by network size), $\tau_{\eta}$ is the recovery time constant of the resource availability (assumed to be $100 \mathrm{~ms}$ ) in the absence of network activity, and the factor of $\eta_{0}(\alpha(t))$ in the denominator ensures that depletion is fast while recovery is slow. Biologically, the hypothesized resource depletion is likely to be a local phenomenon, but the extent of the locality remains unknown. As further experiments reveal the actual distance over which the network could act on a synapse, sub-volumes can be defined and $\eta(t)$ computed for each voxel in the context of the whole network. We, however, did not define sub-volumes of the network and considered the firing of all neurons when computing $\eta(t)$, representing the average resource availability for the entire network volume.

To regulate LTP induced by $\mathrm{STDP}^{+}$events, we scaled the computed synaptic weight change $\Delta W_{+}$by the resource availability $\eta(t)$ and implemented the scaling as

$$
\Delta W_{+}^{\prime}=\gamma(\eta(t)) \cdot \Delta W_{+}
$$

where $\gamma(\eta)$ is a sigmoidal resource modulation function,

$$
\gamma(\eta(t))=\frac{2}{1+\exp \left(\frac{\eta^{*}-\eta(t)}{m}\right)}-1,
$$

with $\eta^{*}=0.6$ defining the LTP-LTD reversal point and $m=0.03$ the steepness of the reversal. In this model, synapses active at the onset of bursts consume resources needed to potentiate, thus forcing later activated synapses to depress.

\section{Criticality Analysis}

We fit a power-law using methods described in Clauset et al. (2009) to the cumulative burst size distribution of the network PSTH ( $\mathrm{dt}=2 \mathrm{~ms}$ ) normalized by the SD of the activity of the sub-threshold network model $(\omega=1.2 \mathrm{nS})$ without plasticity. The threshold for burst detection was set to the mean network firing rate normalized by the SD of the network firing rate when $\omega=1.2 \mathrm{nS}$ plus two. The branching parameter $(\sigma)$ was computed as previously described (Beggs and Plenz, 2003; Priesemann et al., 2009).

\section{Results}

\section{Network-Timing-Dependent Plasticity}

We investigated in acute slices of juvenile rats mounted on a $3 \mathrm{D}$ MEA (Figure 1A) the effect of precisely timed network bursts on STDP at excitatory synaptic inputs to layer 5 pyramidal neurons. STDP protocols known to induce LTP (the STDP ${ }^{+}$ event) and LTD (the STDP $^{-}$event) were applied as a $50 \mathrm{~Hz}$ train of three APs with, respectively, a single evoked EPSP 10 ms earlier or later (Nevian and Sakmann, 2006; Figure 1, see Materials and Methods). The $\mathrm{STDP}^{+}$event reliably induced LTP (Figures 1B,D; $\triangle \mathrm{EPSP}=103 \pm 33 \%, n=11$ ) and the STDP $^{-}$event reliably induced LTD (Figures 1C,D; $\triangle \mathrm{EPSP}=$ $44 \pm 10 \%, n=6$ ), also as previously reported (Nevian and 
Sakmann, 2006). Network bursts were evoked by extra-cellular electrical stimulation of layer 5 using a 3D-MEA (see Materials and Methods). On average, the network burst failed to evoke a spike in $6.8 \%$ of cases, triggered a single spike in $89.2 \%$ of cases, and two spikes in $4 \%$ of cases (Supplementary Figure S1). We never observed a network burst causing more than two spikes in the patched cells. The latency of the first evoked spike was $3.7 \pm 0.2 \mathrm{~ms}$ (range 1.4-10.8 ms, $n=3960$ network bursts recorded in 66 cells). Bursts alone evoked at $0.1 \mathrm{~Hz}$ induced LTD in seven out of nine cells ( $\triangle \mathrm{EPSP}=-41 \pm 12 \%$, $p<4 \mathrm{e}-3$, one sample $t$-test to baseline $0 \%)$, whereas one cell exhibited no significant change $(\triangle \mathrm{EPSP}=19 \pm 20 \%)$, and one cell expressed LTP $(\Delta \mathrm{EPSP}=111 \pm 15 \%)$. Pooling all these cells in a single group led to a relatively high variability, and a skewed distribution (Figure 1D; $\triangle \mathrm{EPSP}=-25 \pm 20 \% ; n=9$ ). Taken together, these results indicate that bursts alone evoked at $0.1 \mathrm{~Hz}$ generally induced LTD, consistent with previous reports for $1 \mathrm{~Hz}$ evocation of population EPSPs in hippocampal slices (Stanton and Sejnowski, 1989; Dudek and Bear, 1992), however, a small sub-population of cells expressing LTP cannot be excluded.

We then examined the interaction between burst-induced LTD and STDP ${ }^{+}$events (Figures $2 \mathrm{~A}-\mathbf{C}$ ). The LTP induced by $\mathrm{STDP}^{+}$remained unchanged when bursts coincided with the $\operatorname{STDP}^{+}$events $(\Delta T=0 ; \Delta \mathrm{EPSP}=109 \pm 64 \%, p=0.17 ; n=8)$, indicating that plasticity driven by relative spike timing in a specific synaptic pathway is unaffected by simultaneous network bursts. This result also indicates that LTD normally induced by such network bursts is blocked by simultaneous $\mathrm{STDP}^{+}$events. However, when bursts preceded the $\mathrm{STDP}^{+}$event by $20 \mathrm{~ms}$, LTP flipped into LTD with a magnitude comparable to that of the burst-induced LTD $(\Delta T=-20 \mathrm{~ms} ; \Delta \mathrm{EPSP}=-21 \pm 7 \%$, $\left.p=3 \mathrm{e}^{-4}, n=7\right)$, indicating that immediately preceding network bursts block $\mathrm{STDP}^{+}$-induced LTP. Conversely, STDP ${ }^{+}$events fail to block LTD induced by preceding bursts. Burst- and $\mathrm{STDP}^{+}$-induced plasticity mutually canceled when bursts were evoked $5 \mathrm{~s}$ before or after $\mathrm{STDP}^{+}$events $(\Delta T= \pm 5000 \mathrm{~ms}$; $\Delta \mathrm{EPSP}=7 \pm 24 \%, p=2.8 \mathrm{e}^{-2} ; n=8$ ) or $50 \mathrm{~ms}$ after the $\mathrm{STDP}^{+}$event $(\Delta T=+50 \mathrm{~ms} ; \Delta \mathrm{EPSP}=8 \pm 21 \%$, $\left.p=9.3 \mathrm{e}^{-3} ; n=14\right)$. All statistical tests comparing each protocol to baseline and to other protocols are provided in Supplementary Table S1.

We next tested for interactions between burst-induced LTD and STDP $^{-}$events (Figures 2D-F). Bursts that were simultaneous, $50 \mathrm{~ms}$ before and $5 \mathrm{~s}$ before or after STDP $^{-}$ events had no cumulative effect on the LTD induced by the $\mathrm{STDP}^{-}$events, indicating that LTD is saturated. This saturation suggests that burst- and STDP ${ }^{-}$-induced LTD share expression mechanisms. Surprisingly, LTD flipped into LTP when bursts immediately followed STDP ${ }^{-}$events $(\Delta T=20 \mathrm{~ms}, 81 \pm 50 \%$, $\left.p=3.5 \mathrm{e}^{-2}, n=9\right)$, indicating that the combination of LTD expression mechanisms induced by both burst and STDP ${ }^{-}$events results in the expression of LTP.

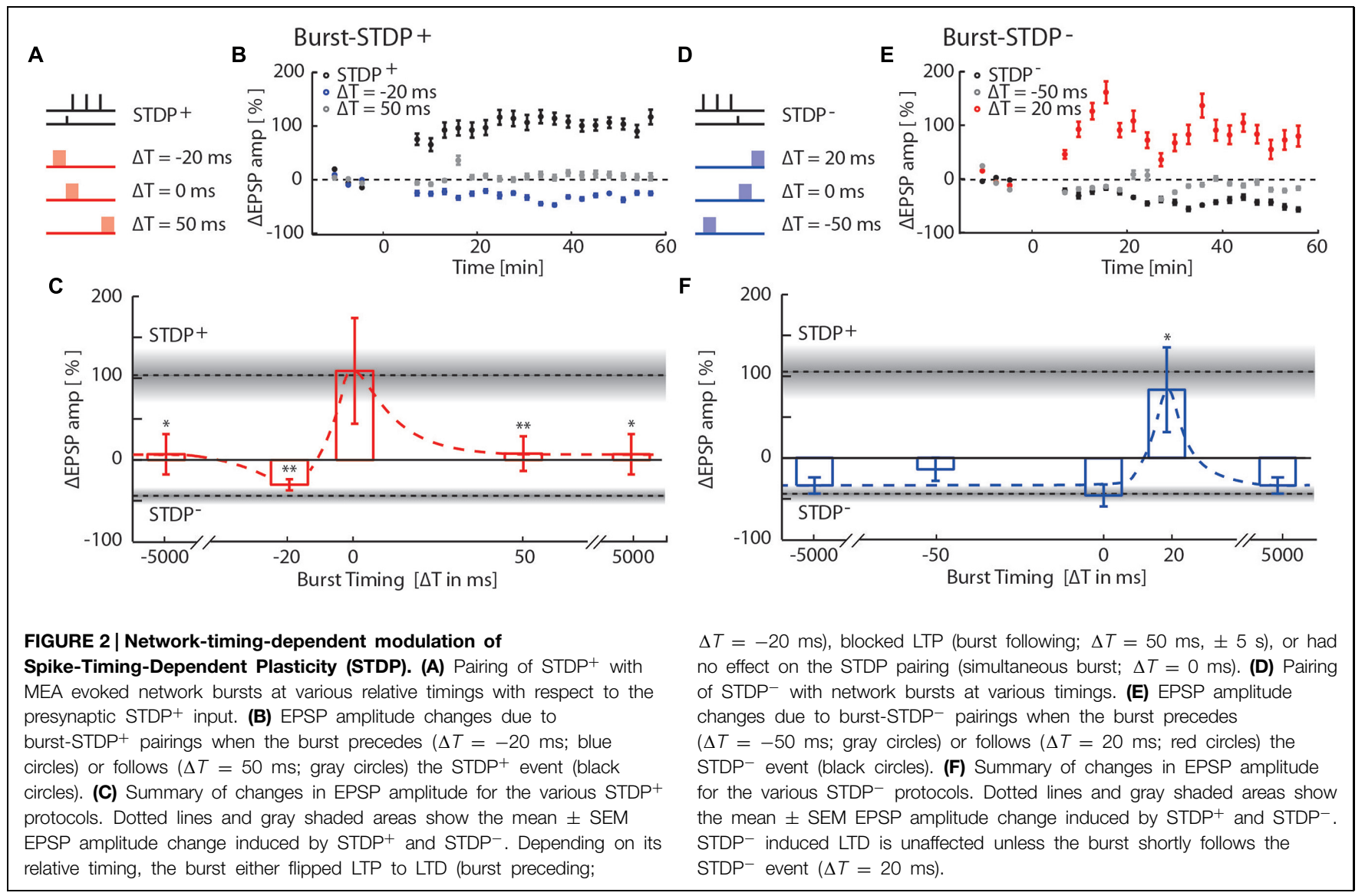


A

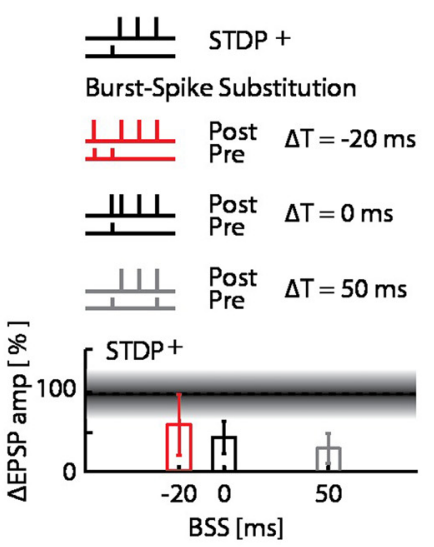

B

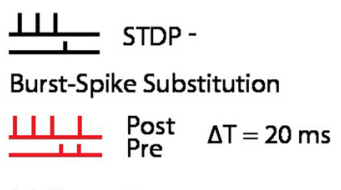

C

... Picrotoxin

- Control

$\sqrt{20 \mathrm{~ms}}$
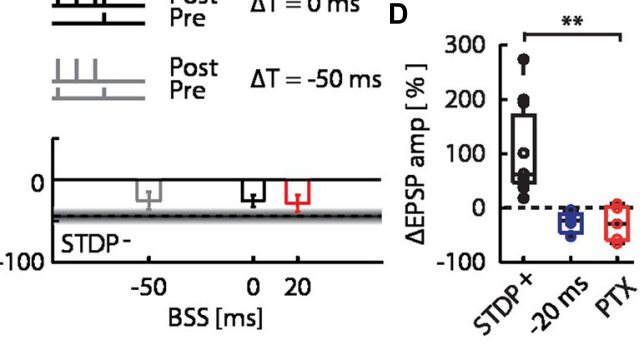

FIGURE 3 | Burst-spike-substitution (BSS) protocols do not explain the flip from LTP to LTD and LTD to LTP, and inhibitory circuits do not contribute to the flip from LTP to LTD. (A) EPSP amplitude changes for BSS protocols of burst-STDP ${ }^{+}$pairings, with simultaneous AP and EPSP at $\Delta T=-20 \mathrm{~ms}$ (red), $\Delta T=0 \mathrm{~ms}$ (black) and $\Delta T=50 \mathrm{~ms}$ (gray). All timings yielded LTP. (B) EPSP amplitude changes for BSS protocols of burst-STDP ${ }^{-}$pairings, with simultaneous AP and EPSP at
$\Delta T=20 \mathrm{~ms}$ (red), $\Delta T=0 \mathrm{~ms}$ (black) and $\Delta T=-50 \mathrm{~ms}$ (gray). All timings yielded LTD. (C) Average normalized EPSP baseline waveforms for control cells, and cells with intracellular picrotoxin (PTX) reveal the effect of PTX on the evoked response by the stimulation with the extracellular pipette. (D) EPSP amplitude change for the STDP ${ }^{+}$event (black) with the network burst at $\Delta T=-20 \mathrm{~ms}$ (blue), and with the network burst at $\Delta T=-20$ ms with PTX (red).
The pairing of STDP events with network bursts can influence the plasticity outcome by alterations of the timing relationship in the pre-post spike motif due to the additional spikes, and by changes in context due to the network burst. To determine whether the observed interaction between bursts and STDP events can be explained entirely by the single presynaptic and single post-synaptic spikes added to the STDP pairing protocol by the MEA stimulation, we repeated the burst-STDP pairing experiments substituting the burst with a single EPSP simultaneous to an AP (Figures 3A,B; BSS, see Materials and Methods). In terms of pre- and post-synaptic spiking, this BSS is equivalent to MEA stimulation (see Materials and Methods and Supplementary Figure S1). BSS could not account for the flip of LTP into LTD due to a burst $20 \mathrm{~ms}$ before the $\mathrm{STDP}^{+}$event (Figure 3A; red bar), nor the flip of LTD into LTP due to a burst $20 \mathrm{~ms}$ after the STDP ${ }^{-}$ event (Figure 3B, red bar). All other BSS timings yielded changes in EPSP amplitudes that were consistent with their respective burst-STDP pairings (Figures 3A,B). These data imply that multiple inputs to the neuron from the bursting network are required to induce the observed flips in directionality of plasticity.

Finally, we examined whether inhibitory synaptic inputs activated by the burst played a role in the observed flip of LTP into LTD. We repeated the burst-STDP ${ }^{+}$experiment with the burst $20 \mathrm{~ms}$ before the $\mathrm{STDP}^{+}$event, while blocking inhibitory currents in the patched cells with intracellular picrotoxin (PTX; Figure 3C; Paille et al., 2013). We found that the bursts still flipped the LTP into LTD (Figure 3D; burst at $\Delta T=-20 \mathrm{~ms}+$ PTX; open red circles; $\triangle$ EPSP amp $=-26 \pm 14 \%, p=0.18$ against STDP $^{+}$ and $p=0.38$ against burst $+\mathrm{STDP}^{+}, n=5$ ), indicating that inhibitory inputs do not play a significant role in burst-dependent STDP.

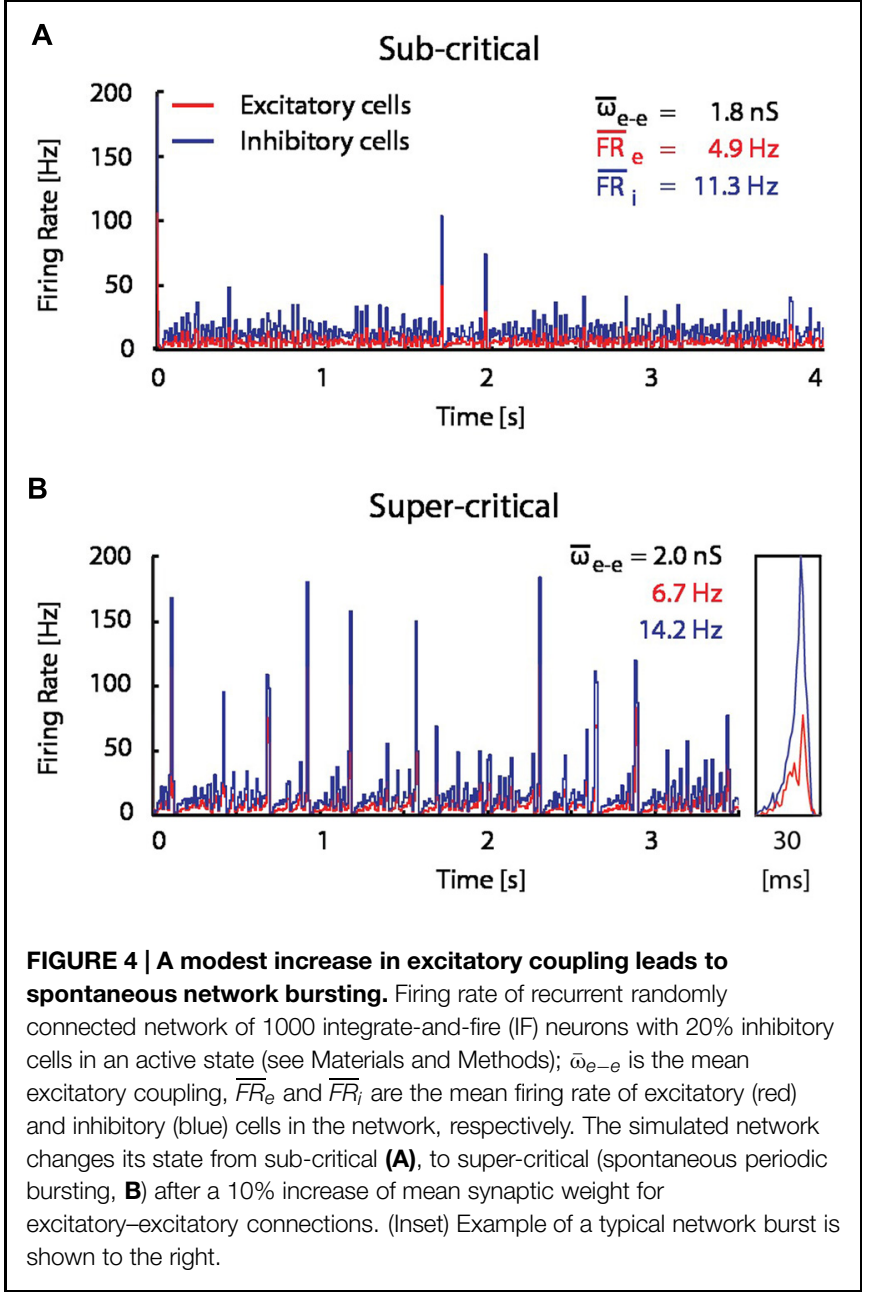


A

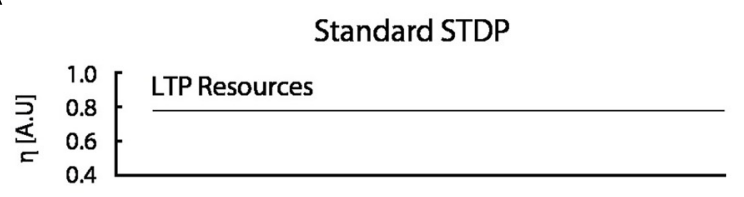

Initial state: sub-critical

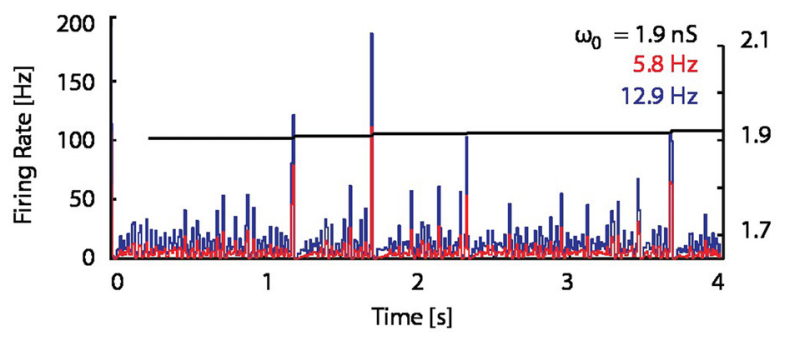

Ending state: supra-critical

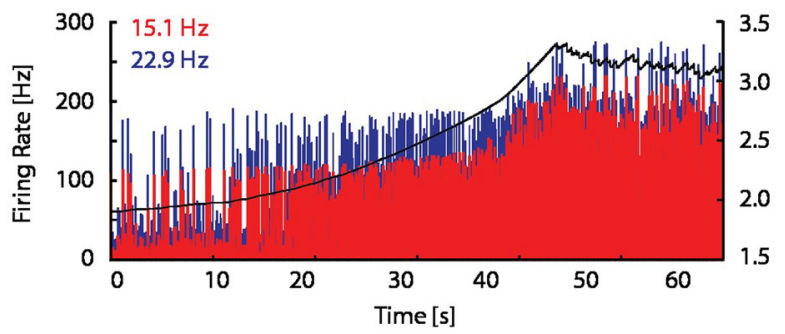

FIGURE 5 |A STDP model with activity-dependent resource consumption counter-balances runaway potentiation. (A) With a standard STDP rule, the firing rate and mean synaptic weight of a network initialized in a sub-critical state drift toward non-physiological
B

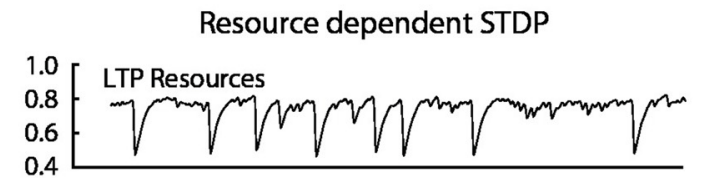

Initial state: supra-critical

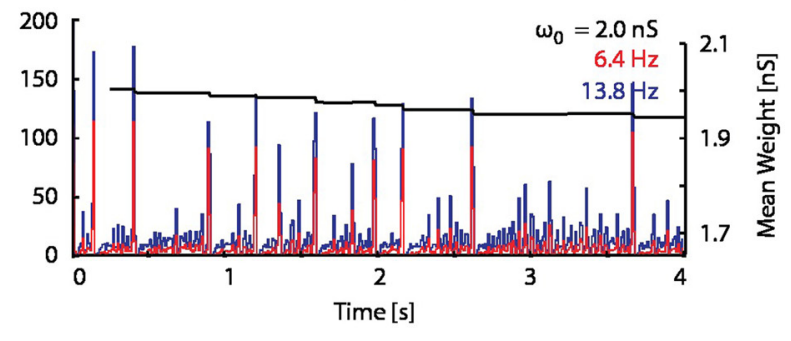

Ending state: critical

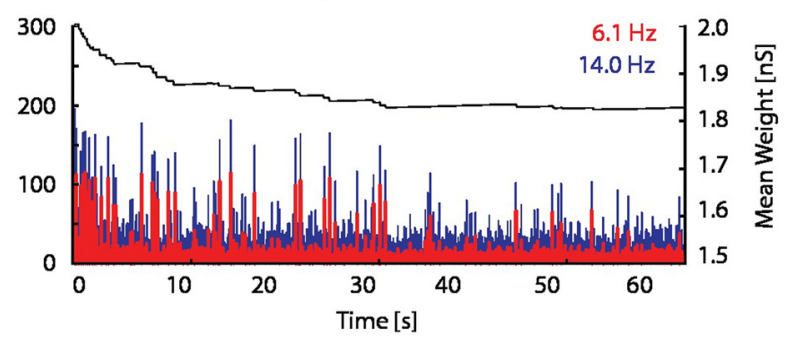

values in a supra-critical state; $\omega_{0}$ is the initial mean excitatory-excitatory synaptic strength. (B) The resource-dependent STDP rule drives networks initialized in a super-critical state toward a critical state.
Taken together, these data suggest that the observed flips of LTD into LTP and LTP into LTD could be manifestations of positive and negative synaptic cooperativity, respectively. We hypothesized that the flip from LTP into LTD (negative cooperativity) could be due to the depletion of critical resources needed for LTP.

\section{Resource-Dependent Regulation of STDP}

In order to assess the significance of the observed flip from LTP into LTD, and the hypothesized resource depletion on STDP, we proposed a resource-dependent STDP learning rule (see Materials and Methods), and examined its implications in network simulations. We simulated the network dynamics and evolution of synaptic weight distributions without STDP, with STDP and with resource-dependent STDP in a simplified network model consisting of 1000 IF neurons ( $80 \%$ excitatory, 20\% inhibitory; see Materials and Methods). Without STDP, network dynamics are highly sensitive to the mean excitatory-excitatory synaptic coupling. For example, a mere $10 \%$ increase in coupling is sufficient to drive the network from a sub-critical regime exhibiting aperiodic occurrence of spontaneous bursts at low frequencies (Figure 4A), to a supra-critical regime exhibiting frequent spontaneous and periodic network bursts (Figure 4B; Beggs and Plenz, 2003; Shew and Plenz, 2013). This transition from sub- to supra-critical activity regimes has been reported in previous theoretical studies (Tsodyks et al., 2000; Kudela et al., 2003) and under pathological experimental conditions where synaptic up-scaling was induced by activity deprivation (Trasande and Ramirez, 2007).

When STDP was introduced into the model network (see Materials and Methods), spontaneous network bursts resulted in more LTP than LTD on average, which gradually increased excitatory-excitatory coupling, and in turn led to an increase in burst frequency and amplitude. This positive feedback drove synaptic weights and network activity to non-physiological regimes (Figure 5A).

When the proposed resource-dependent STDP learning rule was introduced into the model, we found that when networks were initialized with strong excitatory-excitatory coupling that caused supra-critical activity and spontaneous network bursting at low rates, the network converged to a critical level (Figure 5B) in which regular periodic network bursts were replaced with low frequency irregular bursts. Mean synaptic weights also decreased and stabilized at an intermediate value (Figure 5B; bottom panel), consistent with experimental observations (Figure 6A; Bear et al., 1987). On the other hand, the mean synaptic weight for sub-critical networks was found to increase toward 


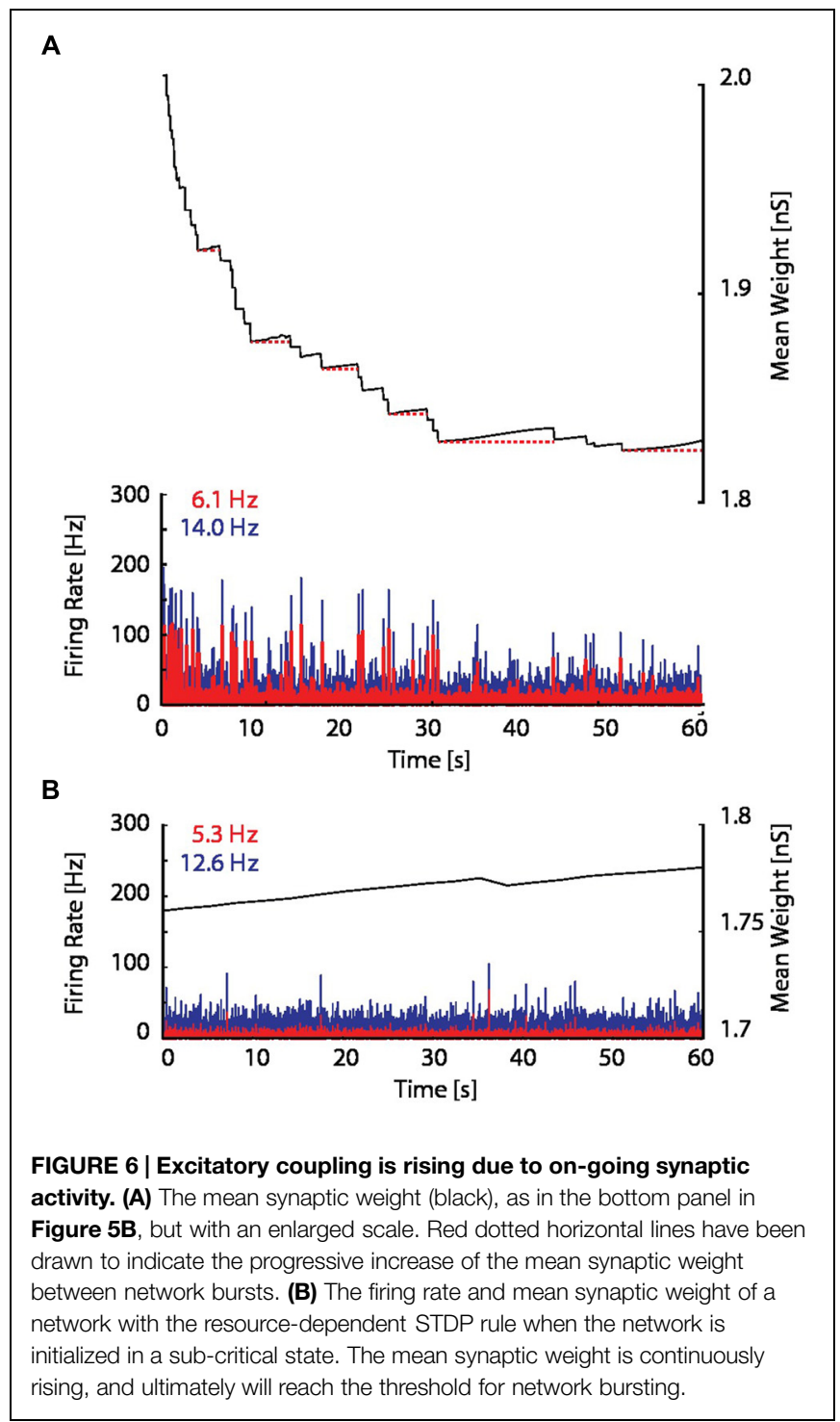

the same intermediate value (Figure 6B). Resource-dependent STDP therefore homeostatically regulates synaptic weights to maintain a mean value just below the threshold for synchronous bursting.

\section{Self-Organized Criticality Emerges from Resource-Dependent STDP}

To determine whether the proposed resource-dependent STDP gives rise to what is known for physical systems as a selforganized critical state (Bak et al., 1988), we analyzed the bursting statistics under the different network conditions described above. Self-organized criticality is indicated when the cumulative probability distribution of event amplitudes follows a power law (Beggs and Plenz, 2003). We therefore plotted the cumulative probability of a burst of a given event size occurring for the network dynamics without STDP, with STDP, and with resource-dependent STDP (Figure 7A). Without STDP and at the implemented mean excitatory-excitatory synaptic couplings $(\omega=1.2,2$, and $3 \mathrm{nS}$ ), the burst-size distribution did not follow a power law distribution (Figure 7A; gray to black dotted lines). With STDP alone, the distribution was markedly different from a power law (Figure 7A; red dotted line). With resource-dependent STDP, all bursts up to 15 times the amplitude of the minimally detected burst followed a power law. The distribution began to deviate when the bursts engaged more than about $20 \%$ of the neurons in the network (largest burst involves around $45 \%$ of the neurons), equivalent to 14 times the mean network activity (Figure 7A; blue dotted line).

Another measure of criticality is the branching parameter (see Materials and Methods), which also gives an indication of the efficiency of the network state to convey information (Beggs and Plenz, 2003; Shew and Plenz, 2013). Networks without STDP have a fixed branching parameter (Figure 7B; gray lines). Networks with STDP alone transitioned to a supra-critical state with a branching parameter greater than 1 (Figure 7B; red line), and were equivalent to networks without STDP and strong excitatory-excitatory synaptic coupling (Figure 7B; solid black line). With resource-dependent STDP, networks converged to a state with a branching parameter around 1, indicating a critical state (Figure $\mathbf{7 B}$; blue line).

\section{Discussion}

Spike-Timing-Dependent Plasticity provides a mechanism to modify the synaptic weight of inputs to a neuron according to their relative timing with respect to the back-propagating AP. We report here a phenomenon we refer to as networktiming-dependent plasticity (NTDP), whereby local spike-timingdependent plasticity of individual synaptic pathways is regulated by the relative timing of synchronous bursts generated by the network. NTDP can regulate STDP by blocking (acting in the opposite plasticity direction), saturating (acting in the same direction) and flipping (acting in the same or opposite direction and crossing a threshold of interaction) depending on the relative timing of synchronous network activity. Positive cooperativity (flipping LTD into LTP) could be explained by cooperative interactions between weak and strong inputs (Levy and Steward, 1983; Sjöström et al., 2001), multiple input-driven facilitation of the bAP (Sjöström and Häusser, 2006) or by the threshold accumulation of resources (same directions of plasticity), such as intracellular calcium levels (Lisman, 1989; Shouval et al., 2002; Graupner and Brunel, 2010, 2012). In the case of the latter, calcium influxes of individual events would not cross the threshold concentration for LTP and consequently lead to depression, but together more easily cross a threshold concentration for LTP induction (Lisman, 1989). Such a hypothesized positive cooperativity would, however, require a temporal separation to explain why LTD was not flipped into LTP when the burst- and $\mathrm{STDP}^{-}$-induced LTD events occurred simultaneously. We further proposed that negative cooperativity (flipping LTP into LTD) could be explained by threshold depletion of 
A

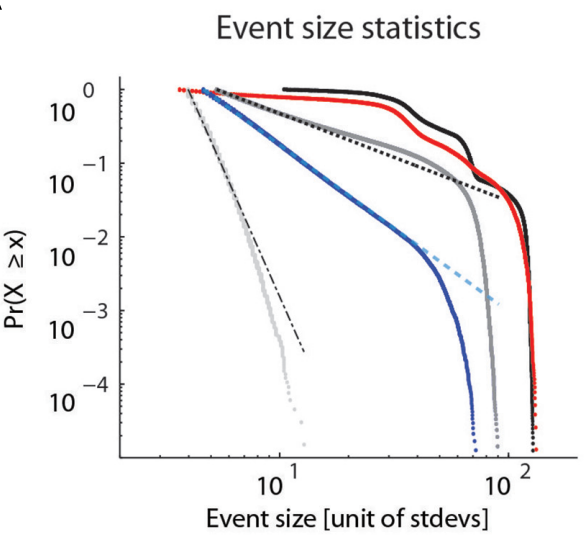

FIGURE 7 | Self-organized criticality emerges from

resource-dependent STDP. (A) Cumulative probability distribution of network burst magnitudes (Clauset et al., 2009). In networks without STDP, varying the mean synaptic weight of excitatory-excitatory connections $\left(\omega=\bar{\omega}_{e-e}\right)$ results in different activity regimes: sub-critical with rare aperiodic network bursts (light gray, $\omega=1.2 \mathrm{nS}$ ), weakly supra-critical with periodic network bursts at rates around $1-10 \mathrm{~Hz}$ (dark gray, $\omega=2 \mathrm{nS}$ ) or strongly supra-critical with periodic network bursts at high rates (black, $\omega=3 \mathrm{nS}$ ). A standard STDP rule drives the network to a strongly super-critical regime
Branching parameter

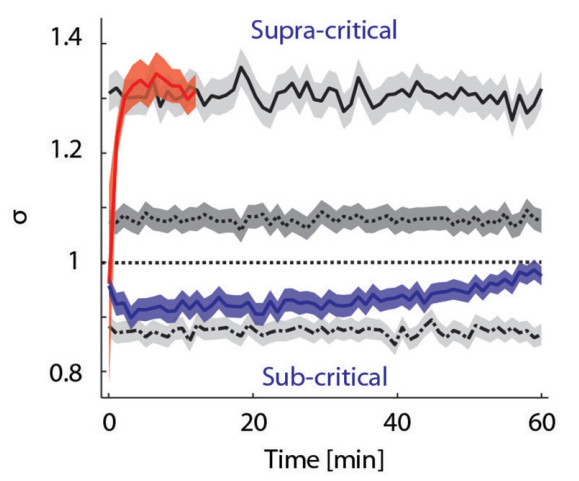

Network without STDP

- $\omega=3 \mathrm{nS}$

- …..... $\omega=2 \mathrm{nS}$

$\cdots \quad \omega=1.2 \mathrm{nS}$

Network with STDP

- _ Standard STDP

- $\ldots$ Resource-STDP

(red), whereas for resource-dependent STDP the resulting burst amplitude statistics follow a power-law (blue). Dashed lines show power-law fits to respective datasets (dot-dashed, $\omega=1.2 \mathrm{nS}$; dashed, $\omega=2 \mathrm{nS}$; cyan-dashed, resource-STDP). (B) The branching parameter $(\sigma)$ is shown for all networks in (a). In networks without STDP, $\sigma$ does not evolve (gray traces, $\omega=1.2,2$, and $3 \mathrm{nS}$ ). With a standard STDP rule, $\sigma$ increases as the network becomes super-critical (red), whereas with the resource-dependent STDP rule a transition from supra-critical to (sub-) critical occurs rapidly and $\sigma$ further converges toward a value around 1 (blue).

resources (opposite directions of plasticity) for LTP, such as extracellular calcium levels (Egelman and Montague, 1999). Together, these data suggest a novel mechanism for embedding local timing rules for synaptic plasticity at individual synaptic pathways into global timing rules for synaptic plasticity in the network.

One caveat of the experimental approach here is that severed neuromodulatory axons remaining in the slices could be evoked to release due to MEA stimulation, and could mediate the observed interactions between the two plasticity induction protocols. Recent advances in optogenetic stimulation methods, which can differentially target pyramidal, inhibitory, and neuromodulatory axon populations, could be employed to clarify their role. Also, applying a standard suite of inhibitors for characterizing the signaling pathways involved could further elucidate the biophysical mechanisms at play and could be pursued in follow-up studies as the basis for more detailed biophysical models.

A model in which the negative cooperativity was implemented as resource-dependent STDP was found to homeostatically regulate synaptic weights in an active network, consistent with previous observations of synaptic down-scaling in disinhibited networks (Turrigiano et al., 1998). Stable biological distributions of synaptic weights are the result, even in the presence of synchronous network activity. Moreover, network burst amplitude statistics were power-law distributed reflecting selforganized criticality, a state optimal for information coding (Bak et al., 1988; Beggs and Plenz, 2003; Shew and Plenz, 2013). Self-organized criticality has been observed in various states of vigilance in vivo (Petermann et al., 2009; Priesemann et al., 2009, 2013; Hahn et al., 2010), but a plasticity rule to achieve and preserve such a state has thus far been missing. The NTDP rule proposed here offers a candidate solution, and may have implications for the mechanisms underlying pathological network states that occur in epilepsy (Trasande and Ramirez, 2007) as well as the down-scaling of synaptic weights during slow-wave sleep (Massimini and Amzica, 2001; Tononi and Cirelli, 2006; Vyazovskiy et al., 2008).

The present experiments indicate that burst- and STDP ${ }^{-}$induced LTD share expression mechanisms. The proposed resource-dependent STDP model accounts for LTD at low frequencies of network bursting (Stanton and Sejnowski, 1989; Dudek and Bear, 1992) while preserving the spike-timing dependence of the underlying STDP rule, thus unifying the two phenomena under one mechanism.

Candidate mechanisms for the observed positive cooperativity rely on variables local to the dendrite (Levy and Steward, 1983; Lisman, 1989; Sjöström et al., 2001; Sjöström and Häusser, 2006). An exploration of the impact of combined negative and positive cooperativity, saturation, and blocking effects on the interaction between burst-induced plasticity and STDP would therefore require simulations of detailed neuron morphology and a biophysical STDP rule. Combined with an experimental characterization of the interactions between STDP and LTP induced by high frequency tetanic stimulation (Bliss and Lomo, 1973; Lynch et al., 1983), this approach could reveal a complement to our proposed resourcedependence of STDP, and provide a unifying model for both directionalities of burst induced plasticity, STDP, and their interactions. 


\section{Author Contributions}

Experiments were conducted by VD. The resource model was designed and implemented by EM, VD, and MP. Network modeling was performed by EM and MP. System criticality analysis was performed by VD and EM. This manuscript has been written by VD, EM, DK, and HM.

\section{Acknowledgments}

We would like to thank W. Gerstner, I. Segev, W. Senn, D. Plenz, V. Priesemann, M.-O. Gewaltig, S. Ramaswamy, G. Chindemi, R.

\section{References}

Bak, P., Tang, C., and Wiesenfeld, K. (1988). Self-organized criticality. Phys. Rev. Lett. 38, 364-374. doi: 10.1103/physreva.38.364

Bear, M. F., Cooper, L. N., and Ebner, F. F. (1987). A physiological basis for a theory of synapse modification. Science 237, 42-48. doi: 10.1126/science.30 37696

Beggs, J. M., and Plenz, D. (2003). Neuronal avalanches in neocortical circuits J. Neurosci. Off. 23, 11167-11177.

Bi, G. Q., and Poo, M. M. (1998). Synaptic modifications in cultured hippocampal neurons: dependence on spike timing, synaptic strength, and postsynaptic cell type. J. Neurosci. Off. 18, 10464-10472.

Bienenstock, E. L., Cooper, L. N., and Munro, P. W. (1982). Theory for the development of neuron selectivity: orientation specificity and binocular interaction in visual cortex. J. Neurosci. 2, 32-48.

Bliss, T. V., and Lomo, T. (1973). Long-lasting potentiation of synaptic transmission in the dentate area of the anaesthetized rabbit following stimulation of the perforant path. J. Physiol. 232, 331-356. doi 10.1113/jphysiol.1973.sp010273

Clauset, A., Shalizi, C. R., and Newman, M. E. J. (2009). Power-law distributions in empirical data. SIAM Rev. 51, 661-703. doi: 10.1137/0707 10111

Coesmans, M., Weber, J. T., De Zeeuw, C. I., and Hansel, C. (2004). Bidirectiona parallel fiber plasticity in the cerebellum under climbing fiber control. Neuron 44, 691-700. doi: 10.1016/j.neuron.2004.10.031

Davison, A. P., Brüderle, D., Eppler, J., Kremkow, J., Muller, E., Pecevski, D., et al. (2009). PyNN: a common interface for neuronal network simulators. Front. Neuroinform. 2:11. doi: 10.3389/neuro.11.011.2008

Destexhe, A., Contreras, D., and Steriade, M. (1998). Mechanisms underlying the synchronizing action of corticothalamic feedback through inhibition of thalamic relay cells. J. Neurophysiol. 79, 999-1016.

Destexhe, A., Rudolph, M., and Paré, D. (2003). The high-conductance state of neocortical neurons in vivo. Nat. Rev. Neurosci. 4, 739-751. doi: $10.1038 / \mathrm{nrn} 1198$

Dudek, S. M., and Bear, M. F. (1992). Homosynaptic long-term depression in area CA1 of hippocampus and effects of N-methyl-D-aspartate receptor blockade. Proc. Natl. Acad. Sci. U.S.A. 89, 4363-4367. doi: 10.1073/pnas.89.1 0.4363

Egelman, D. M., and Montague, P. R. (1999). Calcium dynamics in the extracellular space of mammalian neural tissue. Biophys. J. 76, 1856-1867. doi: 10.1016/S0006-3495(99)77345-5

Fonseca, R., Nägerl, U. V., Morris, R. G. M., and Bonhoeffer, T. (2004). Competing for memory: hippocampal LTP under regimes of reduced protein synthesis. Neuron 44, 1011-1020. doi: 10.1016/j.neuron.2004.10.033

Froemke, R. C., and Dan, Y. (2002). Spike-timing-dependent synaptic modification induced by natural spike trains. Nature 416, 433-438. doi: 10.1038/41 $6433 \mathrm{a}$

Graupner, M., and Brunel, N. (2010). Mechanisms of induction and maintenance of spike-timing dependent plasticity in biophysical synapse models. Front. Comput. Neurosci. 4:136. doi: 10.3389/fncom.2010. 00136
Walker, R. Perin, and S. Muralidhar for comments and helpful discussions. This work was supported by the EPFL Blue Brain Project Fund and the ETH Board Funding to the Blue Brain Project (VD, EM, DK, and HM), the Asterion Foundation (VD), and a Whitaker International Fellowship (MP).

\section{Supplementary Material}

The Supplementary Material for this article can be found online at: http://journal.frontiersin.org/article/10.3389/fncel. 2015.00220/abstract

Graupner, M., and Brunel, N. (2012). Calcium-based plasticity model explains sensitivity of synaptic changes to spike pattern, rate, and dendritic location. Proc. Natl. Acad. Sci. U.S.A. 109, 3991-3996. doi: 10.1073/pnas.11093 59109

Hahn, G., Petermann, T., Havenith, M. N., Yu, S., Singer, W., Plenz, D., et al. (2010). Neuronal Avalanches in Spontaneous Activity In Vivo. J. Neurophysiol. 104, 3312-3322. doi: 10.1152/jn.00953.2009

Hines, M. L., and Carnevale, N. T. (1997). The NEURON simulation environment. Neural Comput. 9, 1179-1209. doi: 10.1162/neco.1997.9. 6.1179

Kudela, P., Franaszczuk, P. J., and Bergey, G. K. (2003). Changing excitation and inhibition in simulated neural networks: effects on induced bursting behavior. Biol. Cybern. 88, 276-285. doi: 10.1007/s00422-002-0381-7

Levy, W. B., and Steward, O. (1983). Temporal contiguity requirements for longterm associative potentiation/depression in the hippocampus. Neuroscience 8, 791-797. doi: 10.1016/0306-4522(83)90010-6

Lisman, J. (1989). A mechanism for the Hebb and the anti-Hebb processes underlying learning and memory. Proc. Natl. Acad. Sci. U.S.A. 86, 9574-9578. doi: 10.1073/pnas.86.23.9574

Lynch, G., Larson, J., Kelso, S., Barrionuevo, G., and Schottler, F. (1983). Intracellular injections of EGTA block induction of hippocampal long-term potentiation. Nature 305, 719-721. doi: 10.1038/305719a0

Markram, H., Lübke, J., Frotscher, M., and Sakmann, B. (1997). Regulation of synaptic efficacy by coincidence of postsynaptic APs and EPSPs. Science 275, 213-215. doi: 10.1126/science.275.5297.213

Markram, H., Gerstner, W., and Sjöström, P. J. (2011). A history of spike-timing-dependent plasticity. Front. Synaptic Neurosci. 3:4. doi: 10.3389/fnsyn.2011.00004

Massimini, M., and Amzica, F. (2001). Extracellular calcium fluctuations and intracellular potentials in the cortex during the slow sleep oscillation. J. Neurophysiol. 85, 1346-1350.

Morrison, A., Aertsen, A., and Diesmann, M. (2007). Spike-timing-dependent plasticity in balanced random networks. Neural Comput. 19, 1437-1467. doi: 10.1162/neco.2007.19.6.1437

Muller, E., Buesing, L., Schemmel, J., and Meier, K. (2007). Spike-frequency adapting neural ensembles: beyond mean adaptation and renewal theories. Neural Comput. 19, 2958-3010. doi: 10.1162/neco.2007.19.11.2958

Nevian, T., and Sakmann, B. (2006). Spine $\mathrm{Ca}^{2+}$ signaling in spiketiming-dependent plasticity. J. Neurosci. 26, 11001-11013. doi 10.1523/JNEUROSCI.1749-06.2006

Paille, V., Fino, E., Du, K., Morera-Herreras, T., Perez, S., Kotaleski, J. H., et al. (2013). GABAergic circuits control spike-timing-dependent plasticity. J. Neurosci. 33, 9353-9363. doi: 10.1523/JNEUROSCI.5796-1 2.2013

Petermann, T., Thiagarajan, T. C., Lebedev, M. A., Nicolelis, M. A. L., Chialvo, D. R., and Plenz, D. (2009). Spontaneous cortical activity in awake monkeys composed of neuronal avalanches. Proc. Natl. Acad. Sci. U.S.A. 106, $15921-$ 15926. doi: 10.1073/pnas.0904089106

Priesemann, V., Munk, M., and Wibral, M. (2009). Subsampling effects in neuronal avalanche distributions recorded in vivo. BMC Neurosci. 10:40. doi: 10.1186/1471-2202-10-40 
Priesemann, V., Valderrama, M., Wibral, M., and Quyen, M. L. V. (2013). Neuronal avalanches differ from wakefulness to deep sleep - evidence from intracranial depth recordings in humans. PLoS Comput. Biol. 9:e1002985. doi: 10.1371/journal.pcbi.1002985

Shew, W. L., and Plenz, D. (2013). The functional benefits of criticality in the cortex. Neuroscientist 19, 88-100. doi: 10.1177/1073858412445487

Shouval, H. Z., and Kalantzis, G. (2005). Stochastic properties of synaptic transmission affect the shape of spike time-dependent plasticity curves. J. Neurophysiol. 93, 1069-1073. doi: 10.1152/jn.00504.2004

Shouval, H. Z., Bear, M. F., and Cooper, L. N. (2002). A unified model of NMDA receptor-dependent bidirectional synaptic plasticity. Proc. Natl. Acad. Sci. U.S.A. 99, 10831-10836. doi: 10.1073/pnas.152343099

Sjöström, P. J., and Häusser, M. (2006). A cooperative switch determines the sign of synaptic plasticity in distal dendrites of neocortical pyramidal neurons. Neuron 51, 227-238. doi: 10.1016/j.neuron.2006.06.017

Sjöström, P. J., Turrigiano, G. G., and Nelson, S. B. (2001). Rate, timing, and cooperativity jointly determine cortical synaptic plasticity. Neuron 32, 11491164. doi: 10.1016/S0896-6273(01)00542-6

Stanton, P. K., and Sejnowski, T. J. (1989). Associative long-term depression in the hippocampus induced by hebbian covariance. Nature 339, 215-218. doi: $10.1038 / 339215 \mathrm{a} 0$

Tononi, G., and Cirelli, C. (2006). Sleep function and synaptic homeostasis. Sleep Med. Rev. 10, 49-62. doi: 10.1016/j.smrv.2005.05.002

Trasande, C. A., and Ramirez, J.-M. (2007). Activity deprivation leads to seizures in hippocampal slice cultures: is epilepsy the consequence of homeostatic plasticity? J. Clin. Neurophysiol. 24, 154-164. doi: 10.1097/WNP.0b013e318033787f
Tsodyks, M., Uziel, A., and Markram, H. (2000). Synchrony generation in recurrent networks with frequency-dependent synapses. J. Neurosci. 20:RC50.

Turrigiano, G. G., Leslie, K. R., Desai, N. S., Rutherford, L. C., and Nelson, S. B. (1998). Activity-dependent scaling of quantal amplitude in neocortical neurons. Nature 391, 892-896. doi: 10.1038/36103

Vyazovskiy, V. V., Cirelli, C., Pfister-Genskow, M., Faraguna, U., and Tononi, G. (2008). Molecular and electrophysiological evidence for net synaptic potentiation in wake and depression in sleep. Nat. Neurosci. 11, 200-208. doi: 10.1038/nn2035

Wiest, M. C., Eagleman, D. M., King, R. D., and Montague, P. R. (2000). Dendritic spikes and their influence on extracellular calcium signaling. J. Neurophysiol. 83, 1329-1337.

Zenke, F., Hennequin, G., and Gerstner, W. (2013). Synaptic plasticity in neural networks needs homeostasis with a fast rate detector. PLoS Comput. Biol. 9:e1003330. doi: 10.1371/journal.pcbi.1003330

Conflict of Interest Statement: The authors declare that the research was conducted in the absence of any commercial or financial relationships that could be construed as a potential conflict of interest.

Copyright (c) 2015 Delattre, Keller, Perich, Markram and Muller. This is an openaccess article distributed under the terms of the Creative Commons Attribution License (CC BY). The use, distribution or reproduction in other forums is permitted, provided the original author(s) or licensor are credited and that the original publication in this journal is cited, in accordance with accepted academic practice. No use, distribution or reproduction is permitted which does not comply with these terms. 